\title{
Abnormal organization of white matter networks in patients with subjective cognitive decline and mild cognitive impairment
}

\author{
Xiao-Ni Wang ${ }^{1, *}$, Yang Zeng ${ }^{2, *}$, Guan-Qun Chen ${ }^{1}$, Yi-He Zhang ${ }^{2}$, Xuan-Yu Li ${ }^{1}$, Xu- \\ Yang Hao ${ }^{2}$, Yang Yu ${ }^{1}$, Meng Zhang ${ }^{2}$, Can Sheng ${ }^{1}$, Yu-Xia Li ${ }^{1}$, Yu Sun ${ }^{1}$, Hong-Yan Li ${ }^{1}$, \\ Yang Song ${ }^{1}$, Kun-Cheng $\mathrm{Li}^{3}$, Tian-Yi Yan${ }^{2}$, Xiao-Ying Tang ${ }^{2}$ and Ying Han ${ }^{1,4}$ \\ 1 Department of Neurology, XuanWu Hospital of Capital Medical University, Beijing, China \\ ${ }^{2}$ School of Life Science, Beijing Institute of Technology, Beijing, China \\ ${ }^{3}$ Department of Radiology, XuanWu Hospital of Capital Medical University, Beijing, China \\ ${ }^{4}$ Center of Alzheimer's Disease, Beijing Institute for Brain Disorders, Beijing, China \\ * These authors have contributed equally to this work \\ Correspondence to: Ying Han, email: 13621011941@163.com \\ Xiao-Ying Tang, email: xiaoying@bit.edu.cn \\ Tian-Yi Yan, email: yantianyi@bit.edu.cn \\ Keywords: subjective cognitive decline, amnestic mild cognitive impairment, network, white matter, diffusion tensor imaging, \\ Pathology Section \\ Received: October 31,2015 Accepted: June 29,2016 Published: July 13, 2016
}

\section{ABSTRACT}

Network analysis has been widely used in studying Alzheimer's disease (AD). However, how the white matter network changes in cognitive impaired patients with subjective cognitive decline (SCD) (a symptom emerging during early stage of $A D$ ) and amnestic mild cognitive impairment (aMCI) (a pre-dementia stage of AD) is still unclear. Here, structural networks were constructed respectively based on FA and FN for 36 normal controls, 21 SCD patients, and 33 aMCI patients by diffusion tensor imaging and graph theory. Significantly lower efficiency was found in aMCI patients than normal controls (NC). Though not significant, the values in those with SCD were intermediate between aMCI and NC. In addition, our results showed significantly altered betweenness centrality located in right precuneus, calcarine, putamen, and left anterior cingulate in aMCI patients. Furthermore, association was found between network metrics and cognitive impairment. Our study suggests that the structural network properties might be preserved in SCD stage and disrupted in aMCI stage, which may provide novel insights into pathological mechanisms of AD.

\section{INTRODUCTION}

Alzheimer's disease (AD), a progressive neurodegenerative disease characterized by memory or other cognitive domain impairments, is the most common type of dementia. Mild cognitive impairment (MCI) is a transient stage between dementia and normal aging. Amnestic MCI (aMCI), a sub-type of MCI, is regarded as a prodromal state of $\mathrm{AD}$ with a dementia conversion rate of $10-15 \%$ [1]. Unfortunately, there is no therapeutic drug to stop or reverse disease progression, but early intervention may slow down the progression of the disease [2].

Subjective cognitive decline (SCD) refers to those who have complains about decline in memory or other cognitive functions, but perform normally on cognitive screening [3]. Recent investigations also demonstrated that SCD patients show a greater risk developing into MCI or dementia and could predict AD independently [4-7]. Amyloid deposition and cerebrospinal fluid AD profile have been found in SCD patients, indicating that AD accounts for a major of SCD. Similar structural or functional alterations have been found in $\mathrm{SCD}$ as $\mathrm{AD}$ or MCI patients [8-16]. Thus, people with SCD are at higher risk of $\mathrm{AD}$ and might be important in the study and early diagnosis for AD.

Diffusion tensor imaging (DTI) is able to noninvasively measure white matter integrity and fiber connectivity in vivo. Previous studies demonstrated widespread white matter impairment (including frontal, 
Table 1: results of demographic characteristics and neuropsychological test

\begin{tabular}{|l|l|l|l|l|l|}
\hline Characteristic & $\mathbf{N C}(\mathbf{n}=\mathbf{3 6})$ & $\mathbf{S C D}(\mathbf{n}=\mathbf{2 1})$ & $\mathbf{a M C I}(\mathbf{n}=\mathbf{3 3})$ & Test statistic & $\boldsymbol{P}$ value \\
\hline Age(y) & $61.8 \pm 7.5$ & $62.9 \pm 9.2$ & $64.3 \pm 9.7$ & $\mathrm{~F}=0.72$ & 0.488 \\
\hline Education(y) & $11.3 \pm 4.4$ & $10.7 \pm 4.1$ & $9.3 \pm 3.7$ & $\mathrm{~F}=1.97$ & 0.145 \\
\hline Sex(M/F) & $12 / 24$ & $6 / 15$ & $16 / 17$ & $\mathrm{x} 2=2.80$ & 0.246 \\
\hline MMSE & $28.1 \pm 1.9$ & $27.9+1.5$ & $25.0 \pm 3.0$ & $\mathrm{~F}=18.36$ & $<0.001 \mathrm{bc}$ \\
\hline MoCA & $26.7 \pm 2.7$ & $26.0 \pm 2.0$ & $19.8 \pm 3.8$ & $\mathrm{~F}=50.64$ & $<0.001 \mathrm{bc}$ \\
\hline AVLT-I & $8.9 \pm 1.5$ & $7.8 \pm 1.9$ & $5.7 \pm 1.5$ & $\mathrm{~F}=33.84$ & $<0.001 \mathrm{abc}$ \\
\hline AVLT-D & $10.3 \pm 2.4$ & $7.9 \pm 2.4$ & $3.9 \pm 2.4$ & $\mathrm{~F}=61.25$ & $<0.001 \mathrm{abc}$ \\
\hline AVLT-R & $12.5 \pm 2.1$ & $10.4 \pm 2.2$ & $7.9 \pm 4.1$ & $\mathrm{~F}=20.72$ & $<0.001 \mathrm{abc}$ \\
\hline
\end{tabular}

Values are given as mean $\pm \mathrm{SD}$

${ }^{a} \mathrm{MCI}$ : amnestic mild cognitive impairment; SCD: subjective cognitive decline; NC:normal control; MMSE: Mini-Mental State Examination; MoCA: Montreal Cognitive Assessment; AVLT: Auditory Verbal Learning Test;

${ }^{a}$ normal control group and SCD group showed significant differences $(\mathrm{P}<0.05)$

${ }^{\mathrm{B}}$ normal control group and aMCI group showed significant differences $(\mathrm{P}<0.05)$

${ }^{c} \mathrm{SCD}$ group and aMCI group showed significant differences $(\mathrm{P}<0.05)$

parietal, temporal lobes, the corpus callosum) in both MCI and SCD patients [12, 17-21]. While a growing body of evidence emerging from various techniques suggested that $\mathrm{AD}$ is a disconnection syndrome and a failure of dynamic network [22-24]. Network analysis based on the white matter network in $\mathrm{AD}$ have suggested that both patients and controls present small-world characteristics with high local inter-connectivity and small path lengths, but implied a weakening small-worldness with either abnormal local efficiency or global efficiency in patients [25-30]. Disrupted topological properties of structural network was also found in preclinical $\mathrm{AD}$ as compared to normal controls [31]. While how small-word properties change in SCD and aMCI patients as well as the correlation between the alterations and behavior are not clear.

Here, we used DTI tractography and graph theory to construct weighted structural networks for the NC, $\mathrm{SCD}$, and aMCI groups respectively. Then, both global and nodal parameters were compared among these three groups. We hypothesized that network topographical structure might have been disrupted in SCD stage and become more severe in aMCI stage. We hope to provide new insights into $\mathrm{AD}$ pathological mechanism and early diagnosis.

\section{RESULTS}

\section{Demographics and neuropsychological test results}

Thirty-six normal controls, 21 SCD patients, and 33 aMCI patients were finally included in this study. $4 \mathrm{aMCI}, 2 \mathrm{SCD}$ and 4 normal controls were excluded due to the failure of image registration from T1-weighted image to MNI template. There were no differences in age, education, and gender among the three groups (all $\mathrm{P}>0.05$ ). The Mini-Mental State Examination (MMSE) and Montreal Cognitive Assessment (MoCA) scores were significantly lower in the aMCI group than normal controls (NC) or the SCD group. The group effects in Auditory Verbal Learning Test (AVLT) scores were significant, with the best performance in $\mathrm{NC}$, intermediate performance in SCD patients, and worst performance in aMCI patients. (Table 1)

\section{Global topology of the white matter connectome}

All three groups presented small-world organization (Figure 1). ANCOVAs showed significant group effects on characteristic path length, global efficiency, and local efficiency, with significant decreased global $(\mathrm{P}=0.002)$ and local $(\mathrm{P}=0.007)$ efficiency, and increased characteristic path length $(\mathrm{P}=0.002)$ between aMCI patients and $\mathrm{NC}$, but not between NC and SCD patients or SCD patients and aMCI patients. In addition, there was a linear trend of these altered network properties across three groups (Figure 1).

\section{Hub regions}

The hub regions are shown in Figure 2. Nine hubs were identified in each groups with six regions in common ( bilateral supplementary motor area ( SMA), putamen (PUT) and thalamus (THA)). The three other regions in NC were the right pecuneus (PCUN), superior frontal gyrus and dorsolateral ( SFGdor) and caudate nucleus (CAU), in SCD were the right PCUN, the left superior parietal gyrus (SPG) and cuneus (CUN), and in aMCI were the bilateral CAU and the right gyrus rectus (REC). 


\section{Differences in betweenness centrality}

We further localized the nodes with changed betweenness centrality among the three groups (Figure $3)$. Regions with significant differences across the three groups were located in right PCUN, PUT, calcarine fissure and surrounding cortex (CAL) and left Anterior cingulate (ACG). Post hoc test showed decrease in PCUN and increase in CAL and PUT in aMCI group than NC group. In addition, decrease in PCUN and increase in ACG and PUT were also found in aMCI as compared to the SCD patients.

\section{Relationship between network metrics and cognition}

We further examined the correlation between network metrics and behavior performance (Figure 3). In SCD patients, no correlation between network properties and neuropsychological tests was found. In the aMCI group, the left ACG was positively correlated with scores of MoCA ( $\mathrm{r}=0.37, \mathrm{P}=0.044)$ and AVLT delayed recall $(\mathrm{r}=0.453, \mathrm{P}=0.012)$. While negative correlation was found between AVLT delayed recall and right PUT in aMCI groups.

\section{DISCUSSION}

We investigated the architecture of white matter networks in patients with SCD and aMCI. The main findings in our study were as follow: 1) small-world topology in both the normal controls and patients, which is in agreement with previous studies [25-30]; 2) increased path length and decreased efficiency in patients; 3) alterations in regional centrality in patients; 4) correlation between network metrics and behavior.

We demonstrated a lower efficiency in aMCI patients compared to $\mathrm{NC}$, which indicates a less optimal organization on patients. A lower global efficiency as well as a higher Lp found in aMCI patients is in line with previous studies [25-29], which indicates the loss of ability to transmit specialized information rapidly among distant brain regions [32]. While the inconsistency observed between $\mathrm{Cp}$ and local efficiency as found in previous studies might be due to the influence of connection strength on $\mathrm{Cp}$ [33-35]. The loss of nodes and a morediffuse impairment pattern would lead to a higher $\mathrm{Cp}[25$, 36]. So both the increment in $\mathrm{Cp}$ and reduction in local efficiency indicated inefficient/ineffective information transmission in aMCI patients. A novel finding of this study was that SCD patients had values intermediate to aMCI and NC, suggesting a similar damage pattern of structure network in SCD but milder than aMCI patients. Such tendency has been found repeatedly in other studies
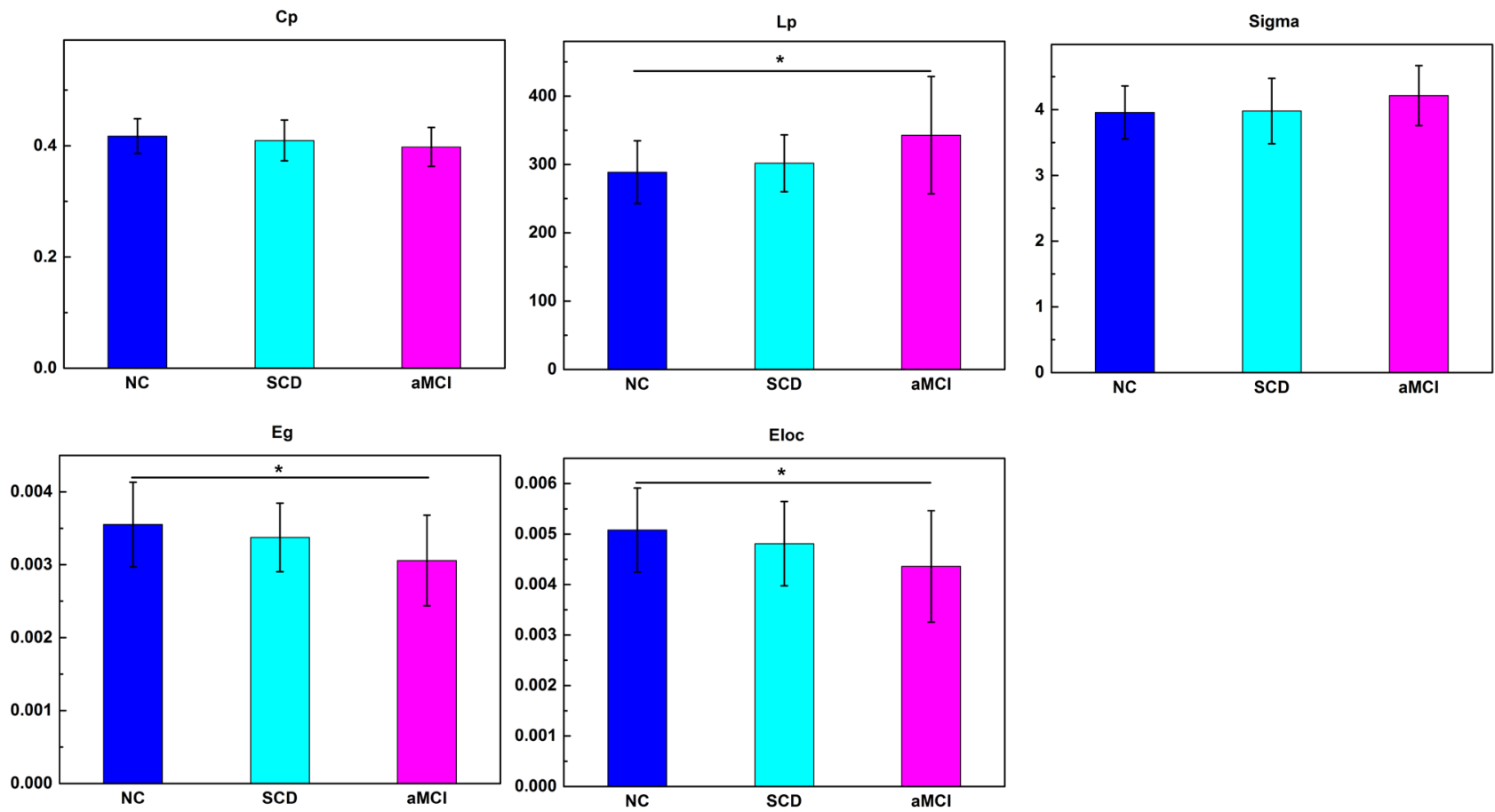

Figure 1: Differences in global measures among the three groups. Significant group effects were observed in characteristic path length, global efficiency and local efficiency. Bars and error bars represent mean values and standard deviations, respectively. $* \mathrm{P}<0.05$. $* * \mathrm{P}<0.01$. NC, normal controls. Eg, global efficiency. Eloc, local efficiency. 
by Tract-Based Spatial Statistics [13].

Recent researches have shown that hubs like the precuneus, the medial frontal cortex, the middle occipital and the cingulated gyrus are more vulnerable $[37,38]$. We found two hubs (right PCUN and putamen) in our study presented significant alterations in centrality with disease prognosis, especially the right PCUN, which was no more a hub in aMCI patients. The PCUN located in medial posterior parietal cortex is a functional core of default mode network (DMN) and involved in various cognitive process like visual-spatial, self-processing, consciousness and episodic memory[39-40]. Decreased nodal strength
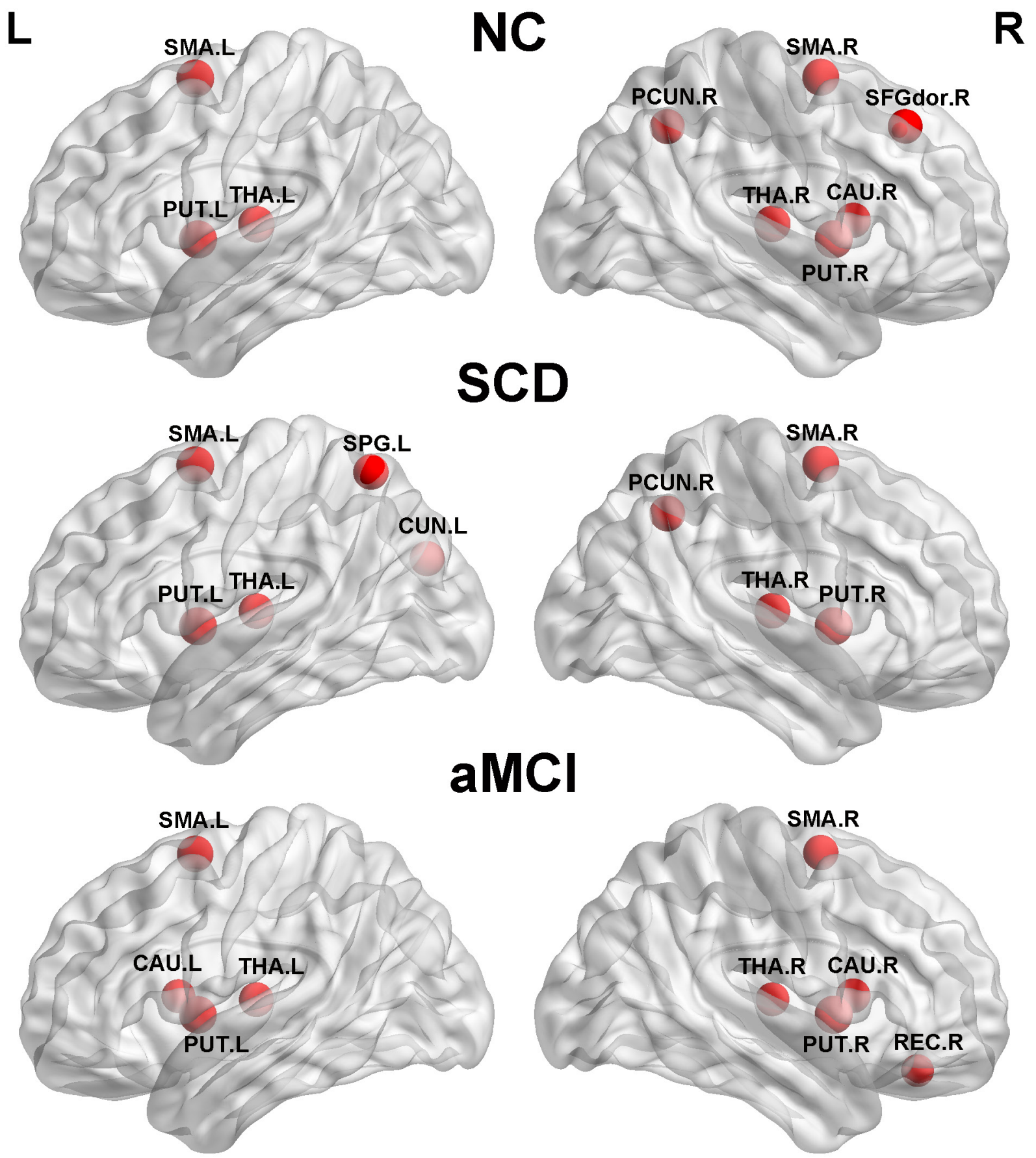

Figure 2: The global network hubs with high betweenness centrality in the normal controls, SCD, and aMCI group. The regions were mapped at a lateral view. SMA, supplementary motor area. PUT, putamen. THA, thalamus. PCUN, precuneus. SFGdor, superior frontal gyrus, dorsolateral. CAU, caudate nucleus. SPG, superior parietal gyrus. CUN, cuneus. REC, gyrus rectus. NC, normal controls. 
was also found in PCUN in previous studies[41]. Evidence also have shown lower FA in PCUN in SCD patients and persons converting to $\mathrm{MCI}[12,42]$. It may provide implication for memory impairment in patients.

The sub-cortical putamen has been found lower nodal strength in aMCI patients in previous studies [41], but showed an increase in centrality in our study conversely. Such alterations were also found in two non-hubs (ACG and calcarine). Betweenness centrality, measuring the importance of the node for information transmission, is not in parallel with node degree [43]. Previous studies have found non-monotonic changes in $\mathrm{AD}$ with higher betweenness centrality in aMCI and mild $\mathrm{AD}$ dementia than prior stages. Increased centrality was also found in lingual gyrus, cingulate and lateral occipitotemporal gyrus by sMRI and fMRI[4445]. We speculated the increase of these nodes might be compensatory for the reduced centrality in PCUN. Further, we found that the centrality in ACG and PUT was correlated with behavior, which indicates the potential
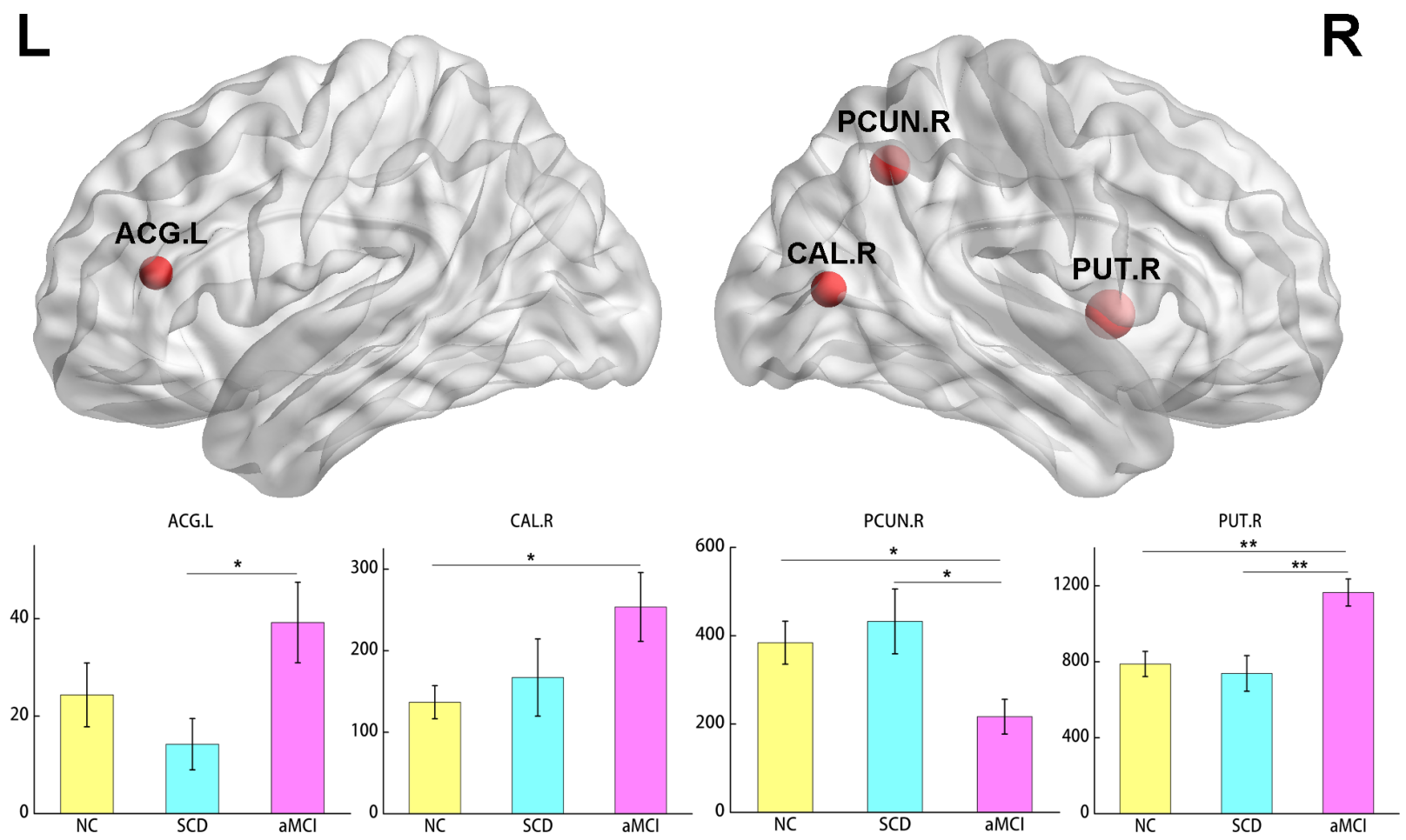

Figure 3: Regions with significant differences in betweenness centrality among the three groups. The node size represents the significance of between-group differences. For each node, the bar and error bar indicate the mean value and standard error, respectively. Post hoc tests showed reduced centrality in PCUN and increased centrality in CAL and PUT in aMCI group versus the NC group. Lower centrality was found in PCUN and higher centrality was found in ACG and PUT in aMCI as compared to the SCD patients. ${ }^{*} \mathrm{P}<0.05$. ${ }^{* *} \mathrm{P}<0.01$. PCUN, precuneus. CAL, calcarine fissure and surrounding cortex. PUT, putamen. ACG: anterior cingulate and paracingulate gyri. NC, normal controls.
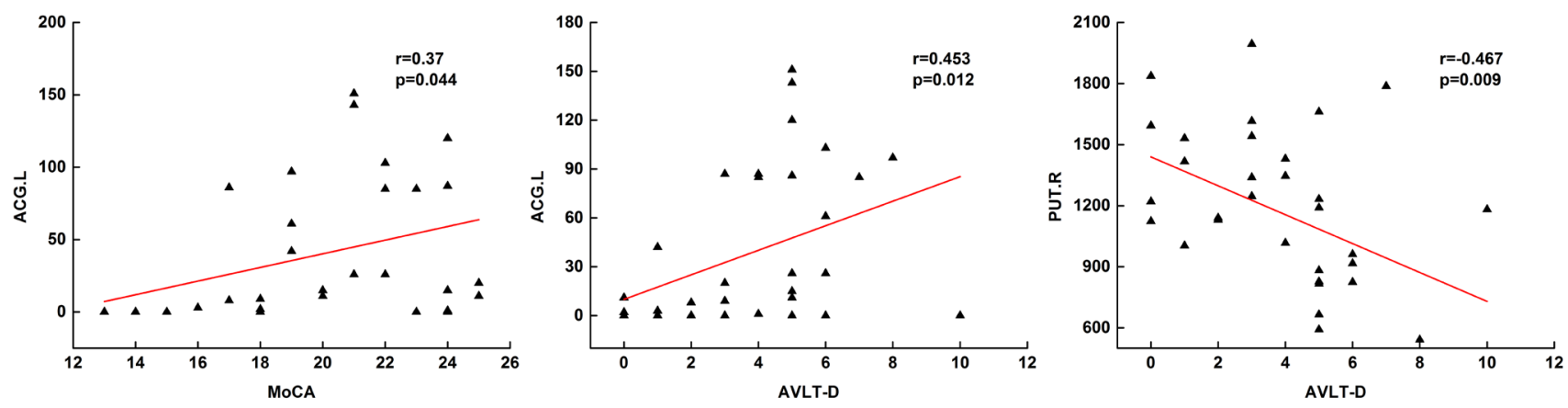

Figure 4: Scatter plots of betweenness centrality and memory performances in aMCI patients. 
of centrality for early diagnosis. While the correlation in ACG was positive, but in PUT was negative. One possible suppose for the discrepancy is that the compensatory mechanism is reserved in PUT with disease progressing but failed for ACG. Despite the aforementioned results, we failed to find any significant difference found between SCD and NC groups, which might because SCD is an earlier stage and the dysfunction is compensatory or because of the limited sample.

We noted that there are still some limitations in our study. First, we employed deterministic tractography to reconstruct structural connectivity as several previous researches [25-27]. While it cannot map out all the fibers accurately, a limitation of tracking crossing fibers and long-distance fibers exists. Second, this study is lack of pathological evidence with PIB-PET or CSF biomarker. Though all subjects went through a series of neuropsychological tests and MRI to exclude other diseases like depression, brain trauma, and vascular dementia, we could not completely ensure that no patients had mixed pathology. Third, this study is crosssectional and a longitudinal study would be valuable to further explore the network properties of AD. Finally, the sample size now is limited and the results need to be replicated in large samples. In conclusion, our results verified that $\mathrm{AD}$ is a disconnection syndrome again. Our findings indicated that white matter network was gradually disrupted as cognitive decline and it has the potential for early diagnosis.

\section{MATERIALS AND METHODS}

\section{Subjects}

A total of 100 right-handed subjects (40 normal controls, $23 \mathrm{SCD}, 37$ aMCI) were recruited at the memory clinic of Beijing XuanWu Hospital of Capital Medical University in China from January 2011 to March 2015. This study was approved by the Medical Research Ethics Committee and Institutional Review Board of XuanWu Hospital (ClinicalTrials.gov Identifier: NCT02353845) and informed consent was obtained from all subjects.

The patients with aMCI were diagnosed according to Petersen's criteria [1] and National Institute on AgingAlzheimer's Association criteria for MCI due to AD [46]as following: (a) memory complaint; (b) objective memory impairment - MMSE, MoCA [47], AVLT; (c) near-normal performance on general cognition and preserved daily life activities; (d) the Clinical Dementia Rating (CDR) score of 0.5; (e) failure to meet the criteria for dementia according to the Diagnostic and Statistical Manual of Mental Disorders, fourth edition, revised (DSM-IV)[48]; (f) hippocampal atrophy observed and (h) the Han nationality, right-handed (the Edinburgh handedness scale score $>40$ points). The patients with SCD were included as following based on the research criteria for subjective cognitive decline [3]: a) selfexperienced decline in memory compared to previous statue (within the last 5 years), which could be confirmed by informants; and (b) normal performances on objective cognitive tests and CDR score $=0$. The normal controls were cognitively normal and had a Clinical Dementia Rating (CDR) of 0. Subjects were excluded if they met the following clinical characteristics: (a) those who have a clear history of stroke (Hachinski Ischemic Scale score (HIS) $>7$ points); (b) severe depression (Hamilton Depression Rating Scale score (HAMD) > 24 points); (c) cognitive impairment caused by traumatic brain injury; (d) other nervous system diseases, which could cause cognitive impairment; (e) systemic diseases, which could cause cognitive impairment; (f) a history of psychosis or congenital mental growth retardation; and $(\mathrm{g})$ those who cannot corporate with neuropsychological tests or have any contraindication to MRI (Magnetic Resonance Imaging). All of subjects underwent MMSE, MoCA, AVLT, Activity of Daily Living (ADL), HIS, HAMD, and CDR. And all participants underwent a brain MRI.

\section{MRI Acquisition}

The MR images of all patients and normal controls were acquired by a Siemens 3T TrioTim MRI system. T1-weighted MR images were obtained by a 3D magnetization-prepared rapid gradient echo (MPRAGE) with following parameters : Slices $=176, \mathrm{TR}=1900$ $\mathrm{ms}, \mathrm{TE}=2.2 \mathrm{~ms}$, inversion time $(\mathrm{TI})=900 \mathrm{~ms}, \mathrm{FA}=9^{\circ}$, field of view $(\mathrm{FOV})=256 \times 256 \mathrm{~mm}$, acquisition matrix $=$ $256 \times 256$, and thickness $=1 \mathrm{~mm}$. DTI data was collected using an echo planar imaging (EPI) sequence with following parameters for three times: in 32 independent, non-collinear directions of a b-value $=1000 \mathrm{~s} / \mathrm{mm} 2$ and one additional image with no diffusion weighting $(b=0)$, slices $=60, \mathrm{TR}=11000 \mathrm{~ms}, \mathrm{TE}=98 \mathrm{~ms}, \mathrm{FA}=90^{\circ}, \mathrm{FOV}$ $=256 \mathrm{~mm} \times 256 \mathrm{~mm}$, acquisition matrix $=128 \times 128$, and thickness $=2 \mathrm{~mm}$.

\section{Image Pre-processing and Network Construction}

Image pre-processing steps contains: Format conversion of original data (DICOM); The extraction of brain tissue and structure; Realignment; Eddy current and motion artifact correction of diffusion-tensor imaging data; Fractional anisotropy calculation; Diffusion tensor tractography. Tractography was conducted to produce 3-D streamlines representing fiber tract connectivity [49].

WM connectivity was modeled as an weighted network containing 90 nodes, defined by automated anatomic labeling [50]. Each AAL brain region was deemed a node of the brain network. With the usage of 
PANDA software, we completed the WM deterministic fiber tracking with a FA threshold of 0.2 and turning angle threshold of 45 degrees of FACT algorithm [49, 51].To limit false positive connections, pairs of nodes were linked if they were interconnected through a certain number of streamlines. In our study, we chose 3 for the threshold value for the streamline number, as in several previous studies [27, 52]. We validated the results setting the threshold ranged from 1 to 5 and found the threshold did not significantly effect the results (see Supplementary Materials).

We computed the weight of each effective connection between two nodes ( $i$ and $j)$ as the product of the connecting fiber number (FN) and mean fractional anisotropy (FA) of the connecting fiber, normalized by the average volume of the two connecting regions to offset the deviation where larger cortical regions are more likely to contain more 'false' fibers $\left(W_{j}=F N^{*} F A /\right.$ vol une ). This weighting method have been employed in several previous diffusion brain network studies [53-54]. Finally, we obtained a symmetric $90 \times 90$ matrix for each participant from their DTI data. This part of work were done by PANDA toolbox [55], which is on the base of FSL[56] (http://fsl.fmrib.ox.ac.uk/fsl/fslwiki).

\section{Network parameter calculation}

Several network topological properties were used to characterize WM structure network derived from each participant, including: clustering coefficient $(\mathrm{Cp})$, characteristic path length (Lp), small-worldness(Sigma), local efficiency (Eloc), global efficiency (Eg) and nodal betweenness $\left(\mathrm{B}_{\text {nod }}\right)$ [32]. In this study, we calculated all these parameter metrics using GRETNA v1.2 (http://www. nitrc.org/projects/gretna), which is a graph-theorectical network analysis toolkit.

For a given network $\mathrm{G}$ with $\mathrm{N}$ nodes, the clustering coefficient $(\mathrm{Cp})$ and the characteristic path length(Lp) were defined by Watts and Strogatz [57] as:

$$
\mathrm{Q} p(\mathrm{G})=\frac{1}{N} \sum_{i \in G} \frac{E_{\text {nod }}}{\mathrm{D}_{\mathrm{i}}(\mathrm{i})\left(\mathrm{D}_{\text {nod }}(\mathrm{i})-1\right) / 2}
$$

Where $D_{\text {nod }}(i)$ is the degree of a node i. $E_{i}$ is the number of edges in $G_{i}$, which is the subgraph composed of the adjacent nodes of a node $i$.

$$
\operatorname{Lp}\left(G=\frac{1}{\frac{1}{N N-1)}\left(\sum_{j \neq i \in G} \frac{1}{L_{j}}\right)}\right.
$$

Where $L_{i j}$ is the shortest path length between nodes $i$ and $j$. To figure out the small-worldness parameter, the values of $\mathrm{Cp}$ and $\mathrm{Lp}$ were normalized by compared with those of 100 random networks $\left(\gamma=\mathrm{Cp}^{\text {real }} / \mathrm{Cp}^{\text {rand }}\right.$ and $\lambda=\mathrm{Lp}^{\text {real }} / \mathrm{Lp}^{\text {rand }}, \mathrm{Si} \operatorname{gm} \boldsymbol{A}=\gamma / \lambda$ ).

A network is said to have small-worldness, if it has similar Lp but higher $\mathrm{Cp}$ than random networks. In other words, a small-word network has a normalized clustering coefficient $(\gamma \approx 1)$ and a normalized path length $(\lambda>1)$.

The global efficiency and local efficiency of $\mathrm{G}$ were defined by Latora and Marchiori[58] as:

$$
\operatorname{Eg}\left(G=\frac{1}{N N-1)} \sum_{j \neq \in \in G} \frac{1}{L_{j}}\right.
$$

and

$$
\operatorname{\theta od} \mathrm{G}=\frac{1}{\mathrm{~N}} \sum_{\mathrm{i} \in \mathrm{G}} \mathrm{Eg}(\mathrm{G})
$$

Where $E(G)$ is the global efficiency of $G_{i}$, which is the subgraph consisting of the adjacent nodes of a node $i$.

The nodal matrices measure the importance of all nodes in the network. Betweenness centrality evaluates the contribution of a node on the communication for other nodes. The betweenness centrality of a node i was defined by Freeman [59] as:

$$
\mathrm{B}_{\text {nod }}(\mathrm{i})=\sum_{\mathrm{j} \neq \neq \mathrm{k} \in \mathrm{G}} \frac{\delta_{\mathrm{jk}}(\mathrm{i})}{\delta_{\mathrm{jk}}}
$$

Where $\delta_{\mathrm{jk}}$ is the number of shortest paths from a node $\mathrm{j}$ to a node $\mathrm{k}$, and $\delta_{\mathrm{j} k}(\mathrm{i})$ is the number of shortest paths from a node $\mathrm{j}$ to a node $\mathrm{k}$ via a node $\mathrm{i}$ within the network $\mathrm{G}$.

The top $10 \%$ hubs of nodal betweenness were depicted by Brainnet Viewer toolbox [60].

\section{Statistical Analysis}

Statistical analysis was performed with software SPSS v20.0. Group differences in age, years of education and neuropsychological scores were examined with oneway ANOVA. Post hoc pairwise t test with Bonferroni correction for multiple comparison was performed if ANOVA yielded significant results $(\mathrm{P}<0.05)$. Sex data were examined with a Pearson chi-square test. For group effects in global and regional network measures , comparisons were performed among 3 groups using one-way ANOVA with post hoc pairwise t tests with Bonferroni correction, when $\mathrm{P}<0.05$.

Finally, we investigated the relationship between network metrics and behavior by partial correlation analysis with age, gender and education as covariates. To identify the correlation between neuropsychological test scores with specific brain regions, the nodes with significant group differences were performed.

\section{ACKNOWLEDGMENTS}

This article was funded by National Natural Science Foundation of China (Grant No. 31371007, 81430037, 30970823), Beijing Municipal Science \& Technology Commission (Grant No. Z131100006813022) and National Key Department of Neurology funded by 
Chinese Health and Family Planning Committee

\section{CONFLICTS OF INTERESTS}

The authors declare that there is no conflict of interests.

\section{REFERENCES}

1. Petersen RC. Mild cognitive impairment as a diagnostic entity. Journal of internal medicine. 2004; 256(3):183-194.

2. Sperling RA, Jack CR, Jr. and Aisen PS. Testing the right target and right drug at the right stage. Science translational medicine. 2011; 3(111): $111 \mathrm{~cm} 133$.

3. Jessen F, Amariglio RE, van Boxtel M, Breteler M, Ceccaldi M, Chetelat G, Dubois B, Dufouil C, Ellis KA, van der Flier WM, Glodzik L, van Harten AC, de Leon MJ, McHugh P, Mielke MM, Molinuevo JL, et al. A conceptual framework for research on subjective cognitive decline in preclinical Alzheimer's disease. Alzheimers Dement. 2014; 10(6):844-852.

4. Sun Y, Yang FC, Lin CP and Han Y. Biochemical and Neuroimaging Studies in Subjective Cognitive Decline: Progress and Perspectives. CNS Neurosci Ther. 2015; 21(10):768-75. doi: 10.1111/cns.12395.

5. Mitchell AJ, Beaumont H, Ferguson D, Yadegarfar M and Stubbs B. Risk of dementia and mild cognitive impairment in older people with subjective memory complaints: metaanalysis. Acta psychiatrica Scandinavica. 2014; 130(6):439451 .

6. Jessen F, Wiese B, Bachmann C, Eifflaender-Gorfer S, Haller F, Kolsch H, Luck T, Mosch E, van den Bussche H, Wagner M, Wollny A, Zimmermann T, Pentzek M, Riedel-Heller SG, Romberg HP, Weyerer S, et al. Prediction of dementia by subjective memory impairment: effects of severity and temporal association with cognitive impairment. Archives of general psychiatry. 2010; 67(4):414-422.

7. Ronnlund M, Sundstrom A, Adolfsson R and Nilsson LG. Subjective memory impairment in older adults predicts future dementia independent of baseline memory performance: Evidence from the Betula prospective cohort study. Alzheimers Dement. 2015; 11(11):1385-92. doi: 10.1016/j.jalz.2014.11.006.

8. Saykin AJ, Wishart HA, Rabin LA, Santulli RB, Flashman LA, West JD, McHugh TL and Mamourian AC. Older adults with cognitive complaints show brain atrophy similar to that of amnestic MCI. Neurology. 2006; 67(5):834-842.

9. Peter J, Scheef L, Abdulkadir A, Boecker H, Heneka M, Wagner M, Koppara A, Kloppel S, Jessen F and Alzheimer's Disease Neuroimaging I. Gray matter atrophy pattern in elderly with subjective memory impairment. Alzheimers Dement. 2014; 10(1):99-108.

10. Scheef L, Spottke A, Daerr M, Joe A, Striepens N, Kolsch
H, Popp J, Daamen M, Gorris D, Heneka MT, Boecker H, Biersack HJ, Maier W, Schild HH, Wagner M and Jessen F. Glucose metabolism, gray matter structure, and memory decline in subjective memory impairment. Neurology. 2012; 79(13):1332-1339.

11. Schultz SA, Oh JM, Koscik RL, Dowling NM, Gallagher CL, Carlsson CM, Bendlin BB, LaRue A, Hermann BP, Rowley HA, Asthana S, Sager MA, Johnson SC and Okonkwo OC. Subjective memory complaints, cortical thinning, and cognitive dysfunction in middle-aged adults at risk for AD. Alzheimers Dement. 2015; 1(1):33-40.

12. Selnes P, Fjell AM, Gjerstad L, Bjornerud A, Wallin A, Due-Tonnessen P, Grambaite R, Stenset V and Fladby $\mathrm{T}$. White matter imaging changes in subjective and mild cognitive impairment. Alzheimers Dement. 2012; 8(5 Suppl):S112-121.

13. Wang Y, West JD, Flashman LA, Wishart HA, Santulli RB, Rabin LA, Pare N, Arfanakis K and Saykin AJ. Selective changes in white matter integrity in MCI and older adults with cognitive complaints. Biochimica et biophysica acta. 2012; 1822(3):423-430.

14. Rodda J, Dannhauser T, Cutinha DJ, Shergill SS and Walker Z. Subjective cognitive impairment: functional MRI during a divided attention task. European psychiatry. 2011; 26(7):457-462.

15. Wang Y, Risacher SL, West JD, McDonald BC, Magee TR, Farlow MR, Gao S, O'Neill DP and Saykin AJ. Altered default mode network connectivity in older adults with cognitive complaints and amnestic mild cognitive impairment. Journal of Alzheimer's disease. 2013; 35(4):751-760.

16. Ewers M, Brendel M, Rizk-Jackson A, Rominger A, Bartenstein P, Schuff N, Weiner MW and Alzheimer's Disease Neuroimaging I. Reduced FDG-PET brain metabolism and executive function predict clinical progression in elderly healthy subjects. NeuroImage Clinical. 2014; 4:45-52.

17. Liu J, Yin C, Xia S, Jia L, Guo Y, Zhao Z, Li X, Han Y and Jia J. White matter changes in patients with amnestic mild cognitive impairment detected by diffusion tensor imaging. PloS one. 2013; 8(3):e59440.

18. Defrancesco M, Egger K, Marksteiner J, Esterhammer R, Hinterhuber H, Deisenhammer EA and Schocke M. Changes in white matter integrity before conversion from mild cognitive impairment to Alzheimer's disease. PloS one. 2014; 9(8):e106062.

19. Wai YY, Hsu WC, Fung HC, Lee JD, Chan HL, Tsai ML, Lin YC, Wu YR, Ying L and Wang JJ. Tract-based spatial statistics: application to mild cognitive impairment. BioMed research international. 2014; 2014:713079.

20. Lee SH, Coutu JP, Wilkens P, Yendiki A, Rosas HD, Salat $\mathrm{DH}$ and Alzheimer's disease Neuroimaging I. Tract-based analysis of white matter degeneration in Alzheimer's disease. Neuroscience. 2015; 301:79-89. 
21. Selnes P, Aarsland D, Bjornerud A, Gjerstad L, Wallin A, Hessen E, Reinvang I, Grambaite R, Auning E, Kjaervik VK, Due-Tonnessen P, Stenset V and Fladby T. Diffusion tensor imaging surpasses cerebrospinal fluid as predictor of cognitive decline and medial temporal lobe atrophy in subjective cognitive impairment and mild cognitive impairment. Journal of Alzheimer's disease. 2013; 33(3):723-736.

22. Xie $\mathrm{T}$ and He Y. Mapping the Alzheimer's brain with connectomics. Frontiers in psychiatry. 2011; 2:77.

23. Reid AT and Evans AC. Structural networks in Alzheimer's disease. European neuropsychopharmacology. 2013; 23(1):63-77.

24. Kurakin A and Bredesen DE. Dynamic self-guiding analysis of Alzheimer's disease. Oncotarget. 2015; 6(16):1409214122. doi: 10.18632/oncotarget.4221.

25. Zhang B, Zhang X, Zhang F, Li M, Schwarz CG, Zhang J, Yin Z, Qian L, Zhao H, Wang K, Tian C, Yu H, Chen W, Lu F, Wu W, Yang QX, et al. Characterizing topological patterns in amnestic mild cognitive impairment by quantitative water diffusivity. Journal of Alzheimer's disease. 2015; 43(2):687-697.

26. Bai F, Shu N, Yuan Y, Shi Y, Yu H, Wu D, Wang J, Xia $\mathrm{M}, \mathrm{He} \mathrm{Y}$ and Zhang Z. Topologically convergent and divergent structural connectivity patterns between patients with remitted geriatric depression and amnestic mild cognitive impairment. The Journal of neuroscience. 2012; 32(12):4307-4318.

27. Shu N, Liang Y, Li H, Zhang J, Li X, Wang L, He Y, Wang $\mathrm{Y}$ and Zhang Z. Disrupted topological organization in white matter structural networks in amnestic mild cognitive impairment: relationship to subtype. Radiology. 2012; 265(2):518-527.

28. Lo CY, Wang PN, Chou KH, Wang J, He Y and Lin CP. Diffusion tensor tractography reveals abnormal topological organization in structural cortical networks in Alzheimer's disease. The Journal of neuroscience. 2010; 30(50):1687616885 .

29. Daianu M, Jahanshad N, Nir TM, Toga AW, Jack CR, Jr., Weiner MW, Thompson PM and Alzheimer's Disease Neuroimaging I. Breakdown of brain connectivity between normal aging and Alzheimer's disease: a structural k-core network analysis. Brain connectivity. 2013; 3(4):407-422.

30. Daianu M, Jahanshad N, Nir TM, Jack CR, Jr., Weiner MW, Bernstein MA, Thompson PM and Alzheimer's Disease Neuroimaging I. Rich club analysis in the Alzheimer's disease connectome reveals a relatively undisturbed structural core network. Human brain mapping. 2015; 36(8):3087-3103

31. Fischer FU, Wolf D, Scheurich A, Fellgiebel A and Alzheimer's Disease Neuroimaging I. Altered whole-brain white matter networks in preclinical Alzheimer's disease. NeuroImage Clinical. 2015; 8:660-666.

32. Rubinov M and Sporns O. Complex network measures of brain connectivity: uses and interpretations. NeuroImage. 2010; 52(3):1059-1069.

33. Sanabria-Diaz G, Martinez-Montes E, Melie-Garcia L and Alzheimer's Disease Neuroimaging I. Glucose metabolism during resting state reveals abnormal brain networks organization in the Alzheimer's disease and mild cognitive impairment. PloS one. 2013; 8(7):e68860.

34. Wang R, Wang J, Yu H, Wei X, Yang C and Deng B. Decreased coherence and functional connectivity of electroencephalograph in Alzheimer's disease. Chaos. 2014; 24(3):033136.

35. Pineda-Pardo JA, Garces P, Lopez ME, Aurtenetxe S, Cuesta P, Marcos A, Montejo P, Yus M, HernandezTamames JA, del Pozo F, Becker JT and Maestu F. White matter damage disorganizes brain functional networks in amnestic mild cognitive impairment. Brain connectivity. 2014; 4(5):312-322.

36. Seo EH, Lee DY, Lee JM, Park JS, Sohn BK, Lee DS, Choe YM and Woo JI. Whole-brain functional networks in cognitively normal, mild cognitive impairment, and Alzheimer's disease. PloS one. 2013; 8(1):e53922.

37. Hagmann P, Cammoun L, Gigandet X, Meuli R, Honey CJ, Wedeen VJ and Sporns O. Mapping the structural core of human cerebral cortex. PLoS biology. 2008; 6(7):e159.

38. Gong G, He Y, Chen ZJ and Evans AC. Convergence and divergence of thickness correlations with diffusion connections across the human cerebral cortex. NeuroImage. 2012; 59(2):1239-1248.

39. Cavanna AE and Trimble MR. The precuneus: a review of its functional anatomy and behavioural correlates. Brain. 2006; 129(Pt 3):564-583.

40. Utevsky AV, Smith DV and Huettel SA. Precuneus is a functional core of the default-mode network. The Journal of neuroscience. 2014; 34(3):932-940.

41. Wang J, Zuo X, Dai Z, Xia M, Zhao Z, Zhao X, Jia J, Han $\mathrm{Y}$ and He Y. Disrupted functional brain connectome in individuals at risk for Alzheimer's disease. Biological psychiatry. 2013; 73(5):472-481.

42. Zhuang L, Sachdev PS, Trollor JN, Kochan NA, Reppermund S, Brodaty H and Wen W. Microstructural white matter changes in cognitively normal individuals at risk of amnestic MCI. Neurology. 2012; 79(8):748-754.

43. Li Y, Qin Y, Chen X and Li W. Exploring the functional brain network of Alzheimer's disease: based on the computational experiment. PloS one. 2013; 8(9):e73186.

44. Kim H, Yoo K, Na DL, Seo SW, Jeong J and Jeong Y. Non-monotonic reorganization of brain networks with Alzheimer's disease progression. Frontiers in aging neuroscience. 2015; 7:111.

45. He Y, Chen Z and Evans A. Structural insights into aberrant topological patterns of large-scale cortical networks in Alzheimer's disease. The Journal of neuroscience. 2008; 28(18):4756-4766.

46. Albert MS, DeKosky ST, Dickson D, Dubois B, Feldman 
HH, Fox NC, Gamst A, Holtzman DM, Jagust WJ, Petersen RC, Snyder PJ, Carrillo MC, Thies B and Phelps CH. The diagnosis of mild cognitive impairment due to Alzheimer's disease: recommendations from the National Institute on Aging-Alzheimer's Association workgroups on diagnostic guidelines for Alzheimer's disease. Alzheimers Dement. 2011; 7(3):270-279.

47. Lu J, Li D, Li F, Zhou A, Wang F, Zuo X, Jia XF, Song $\mathrm{H}$ and Jia J. Montreal cognitive assessment in detecting cognitive impairment in Chinese elderly individuals: a population-based study. Journal of geriatric psychiatry and neurology. 2011; 24(4):184-190.

48. Gmitrowicz A and Kucharska A. [Developmental disorders in the fourth edition of the American classification: diagnostic and statistical manual of mental disorders (DSM IV -- optional book)]. [Article in Polish]. Psychiatria polska. 1994; 28(5):509-521.

49. Mori S, Crain BJ, Chacko VP and van Zijl PC. Threedimensional tracking of axonal projections in the brain by magnetic resonance imaging. Annals of neurology. 1999; 45(2):265-269.

50. Tzourio-Mazoyer N, Landeau B, Papathanassiou D, Crivello F, Etard O, Delcroix N, Mazoyer B and Joliot M. Automated anatomical labeling of activations in SPM using a macroscopic anatomical parcellation of the MNI MRI single-subject brain. NeuroImage. 2002; 15(1):273-289.

51. Basser PJ, Pajevic S, Pierpaoli C, Duda J and Aldroubi A. In vivo fiber tractography using DT-MRI data. Magnetic resonance in medicine. 2000; 44(4):625-632.

52. Gong G, He Y, Concha L, Lebel C, Gross DW, Evans AC and Beaulieu C. Mapping anatomical connectivity patterns of human cerebral cortex using in vivo diffusion tensor imaging tractography. Cerebral cortex. 2009; 19(3):524536.

53. Brown JA, Terashima KH, Burggren AC, Ercoli LM, Miller KJ, Small GW and Bookheimer SY. Brain network local interconnectivity loss in aging APOE-4 allele carriers. Proceedings of the National Academy of Sciences of the United States of America. 2011; 108(51):20760-20765.

54. Chen Z, Liu M, Gross DW and Beaulieu C. Graph theoretical analysis of developmental patterns of the white matter network. Frontiers in human neuroscience. 2013; 7:716.

55. Cui Z, Zhong S, Xu P, He Y and Gong G. PANDA: a pipeline toolbox for analyzing brain diffusion images. Frontiers in human neuroscience. 2013; 7:42.

56. Smith SM, Jenkinson M, Woolrich MW, Beckmann CF, Behrens TE, Johansen-Berg H, Bannister PR, De Luca M, Drobnjak I, Flitney DE, Niazy RK, Saunders J, Vickers J, Zhang Y, De Stefano N, Brady JM, et al. Advances in functional and structural MR image analysis and implementation as FSL. NeuroImage. 2004; 23 Suppl 1:S208-219.
57. Watts DJ and Strogatz SH. Collective dynamics of 'smallworld' networks. Nature. 1998; 393(6684):440-442.

58. Latora $\mathrm{V}$ and Marchiori $\mathrm{M}$. Efficient behavior of small-world networks. Physical review letters. 2001; 87(19):198701.

59. Freeman L C. A set of measures of centrality based on betweenness. Sociometry. 1977; 40(1):35-41.

60. Xia M, Wang J and He Y. BrainNet Viewer: a network visualization tool for human brain connectomics. PloS one. 2013; 8(7):e68910. 